\title{
High-resolution longitudinal profile diagnostics for ultralow charges stored in a ring
}

\author{
Alex H. Lumpkin $\odot^{1, *}$ and Kent P. Wootton $\odot^{2}$ \\ ${ }^{1}$ Argonne Associate, Argonne National Laboratory, Lemont, Illinois 60439, USA \\ ${ }^{2}$ Argonne National Laboratory, Lemont, Illinois 60439, USA
}

(Received 26 February 2021; accepted 24 June 2021; published 30 July 2021; corrected 17 August 2021)

\begin{abstract}
Observations of optical synchrotron radiation emitted over millions of passes from a few electrons circulating in the particle accumulator ring at the Advanced Photon Source were performed with a digital CMOS camera and a synchroscan streak camera operating at 117.3 MHz. Discrete changes of integrated counts in the CMOS image region of interest are ascribed to single electron steps in a 10 -s sensor integration time. Circulations of a single electron at 375 and at $425 \mathrm{MeV}$ were demonstrated in the 12-bit digital CMOS camera images. The streak camera operating at the 12 th harmonic of the fundamental revolution frequency at $9.77 \mathrm{MHz}$ was used to measure the bunch length from $0.5-\mathrm{nC}$ circulating charge down to tens of electrons or $<10 \mathrm{aC}$. The measurements were performed with $\sim 8$-ps temporal resolution uniquely at such ultralow charges. These results also serve as a proof-of-principle longitudinal profile diagnostic for the predicted effects of optical stochastic cooling at low charge in commissioning experiments at the Fermilab Integrable Optics Test Accelerator ring.
\end{abstract}

DOI: 10.1103/PhysRevAccelBeams.24.072806

\section{INTRODUCTION}

Since the first identification of optical synchrotron radiation (OSR) emitted from a circulating beam transiting dipolar magnetic fields in a synchrotron ring in the middle of the last century [1], major light source facilities based on storage rings have been constructed for visible, UV, and eventually x-ray research. In parallel, investigators have also used both OSR and x-ray synchrotron radiation as a diagnostic [2] of the electron beam transverse profiles and longitudinal profiles (and phase). For example, experiments were performed in the past 25 years at the three accelerator rings of the Advanced Photon Source (APS) [3-5], the Advanced Light Source [6], and the Stanford Synchrotron Radiation Laboratory [7] using streak cameras in such studies. In these cases, $\mathrm{nC}$ level charges generally were involved in each of the bunches of the fill pattern so singlebunch, single-turn imaging was possible with microchannel plate (MCP) image intensifier technology [3-7]. With equilibrium bunch lengths at the $40-150 \mathrm{ps}$ regime in the rings, streak tube deflection rate/temporal resolution was relaxed to provide the needed time-axis coverage.

\footnotetext{
*1umpkin@fnal.gov
}

Published by the American Physical Society under the terms of the Creative Commons Attribution 4.0 International license. Further distribution of this work must maintain attribution to the author(s) and the published article's title, journal citation, and DOI.
In this paper, we identify and meet the challenge of extending such characterizations to ultralow charges in a ring at even the $\mathrm{aC}$ level by summing OSR signals over millions of turns in our imaging systems. Investigations into the probability of photon emission via the OSR mechanism from single electrons circulating in small storage rings had been done in the past with spatially integrating and light-intensity-monitoring photomultiplier tubes (PMTs) or photodiodes [8-10] and more recently with PMTs and digital CMOS cameras [11,12]. These gave the intensity effect versus stored electron number and transverse size. However, mapping of the longitudinal distribution of photons emitted by a few electrons circulating in a ring has only been done at the 10-ns level $[8,9]$. We note that observations of OSR generated in a ring dipole by circulating beam has been performed for over two decades with $n C$-level charges with a streak camera in the particle accumulator ring (PAR) at the APS [4]. At APS, the linac produces a 10-ns long macropulse of 28 micropulses with 350 -ps spacing (from the S-band thermionic rf gun) for injection into the PAR. Basically, the damping process involves both a fundamental rf system at $9.77 \mathrm{MHz}$ and a 12th harmonic system at $117.3 \mathrm{MHz}$, and the linac pulse train of 10-ns duration is damped in $<500 \mathrm{~ms}$ to a single equilibrium bunch length of $\sim 0.3 \mathrm{~ns}$ at $0.5 \mathrm{nC}$ stored (with both rf systems powered). These values were determined via OSR and a Hamamatsu C5680 streak camera operating with a slow vertical deflection unit with 50-ns temporal axis coverage, temporal resolution of 50 to $100 \mathrm{ps}$, and $<50$-ps trigger jitter values. This vertical sweep unit was triggered at $200 \mathrm{kHz}$. 
However, to investigate longitudinal distributions for ultralow charges, one needs to increase the trigger rate to the $10-\mathrm{MHz}$ level and have synchronous summing of signals over millions of beam revolutions. To achieve these requirements, we have implemented, for the first time for this objective, a synchroscan streak camera that is phase locked to $117.3 \mathrm{MHz}$, the 12th harmonic of the fundamental revolution frequency at $9.77 \mathrm{MHz}$ of the PAR. This provided temporal resolution of about 8 ps on the slowest streak range with a 1500-ps time-axis coverage matched to the expected bunch lengths. In this case electrons circulate at $1 / 12$ th the streak camera vertical deflection frequency so no turns are missed, and the phase locking of the instrument to the radiofrequency (rf) means the camera phase is nearly the same to a few ps over hundreds of seconds. The streak camera photocathode sees OSR every turn for multiple electrons stored, and its phosphor screen integrates the arrival times of the photoelectron events on the deflection axis. The readout CCD sensor accumulates the phosphor image over $33 \mathrm{~ms}$ since the analog image is readout at $30 \mathrm{~Hz}$ and then digitized.

We also obtained digital CMOS camera images for the single-electron emissions averaged over the acquisition for $10 \mathrm{~s}$ on the chip, as demonstrated by the discrete or quantized steps in the integrated intensity of the OSR-based beam image over 97.7 million turns. These data provided the critical online assessment of the number of stored electrons in the ring in parallel with the streak camera studies. These demonstrations (preliminarily reported in [13]) also provide a proof-of-principle longitudinal diagnostic for optical stochastic cooling (OSC) experiments at ultralow charge as proposed in the Integrable Optics Test Accelerator (IOTA) ring at Fermilab $[14,15]$.

\section{EXPERIMENTAL ASPECTS}

\section{A. The accelerators at APS}

The schematic of the linac and PAR is shown in Fig. 1, and the parameters used in these experiments are given in
Table I. The small ring is designed to accumulate charges injected from the S-band linac which can operate at up to a $30-\mathrm{Hz}$ macropulse rate. Usually, the thermionic cathode rf gun is used to generate a short $\sim 10$-ns-long pulse train of 28 micropulses at $2.86 \mathrm{GHz}$ (micropulse spacing is $350 \mathrm{ps}$ ). Operations at a $30-\mathrm{Hz}$ rate allow the generation of $1-1.8 \mathrm{nC}$ per macropulse. This is far more than we need, so initially for the low-charge studies we ran at $2 \mathrm{~Hz}$ and up to five beam profile screens were inserted in the beam line between the accelerating structures to attenuate the number of electrons actually being captured in the PAR. As we stepped in the screens, we compensated for the input signal loss in the digital camera with increased exposure times from $100 \mu \mathrm{s}$ up to $10 \mathrm{~s}$. After injection into the PAR, the synchrotron radiation damping with rf fundamental and 12th harmonic potential wells lead to a coalesced single bunch with equilibrium longitudinal distributions of $\sim 1 \mathrm{~ns}$ (fundamental rf only) and $0.3 \mathrm{~ns}$ (both rf cavities powered), respectively. We then found that the firing of the PAR injection kickers with various kicker voltages gave us a controllable way to reduce the stored beam current. We demonstrated as described in Sec. III an ultralow relative charge monitor operating at 8 orders of magnitude below the Bergoz integrating current transformer (ICT) limits used normally as a beam intensity monitor for $\mathrm{nC}$ down to $\mathrm{pC}$ values $[16,17]$. These experiments were done at 375 and $425 \mathrm{MeV}$ separately.

\section{B. The optical diagnostics}

The OSR was transported from the PAR West dipole magnet source through a quartz window and directed by $\mathrm{Al}$ mirrors through a 1000-mm focal length $(f)$ lens out of the accelerator enclosure up to a shrouded optics table on the mezzanine above [19]. This configuration (Fig. 2) provided access to the detectors in use, the digital CMOS camera and the Hamamatsu C5680 streak camera. (The PMT on the East dipole source was not optimized for these tests, but it did provide some medium-intensity level data.) The OSR

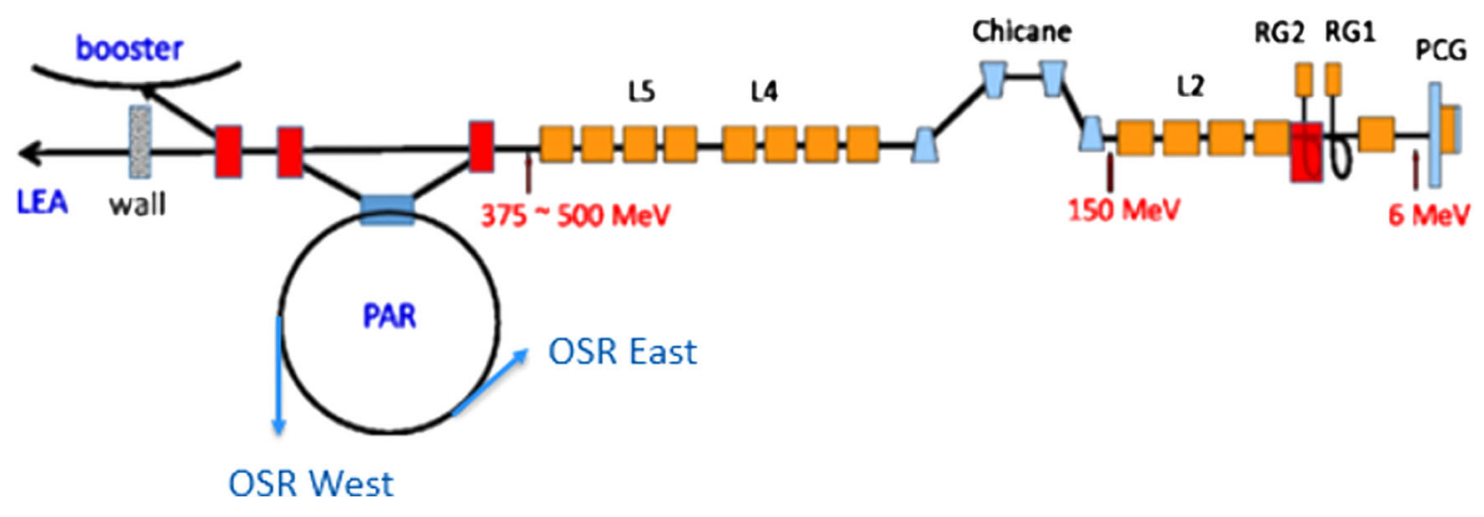

FIG. 1. Schematic layout of the APS S-band linac structures (L\#) with the photocathode gun (PCG), rf thermionic guns (RG1 and $\mathrm{RG} 2$ ), the PAR injector chain, and the optical synchrotron radiation (OSR) ports with optical transport out of the tunnel. The CMOS camera and streak camera use the OSR West source. (Adapted under Creative Commons license CC-BY 4.0 from Ref. [18]). 
TABLE I. Summary of the PAR and electron beam parameters used in the studies.

\begin{tabular}{lcc}
\hline \hline Beam parameter & Units & Value \\
\hline Beam energy & $\mathrm{MeV}$ & $375 ; 425$ \\
Magnetic field & $\mathrm{T}$ & $1.23 ; 1.39$ \\
Revolution frequency & $\mathrm{MHz}$ & 9.77 \\
Stored charge & $\mathrm{pC}$ & $10^{3}$ to $10^{-6}$ \\
Bunch length $(\mathrm{rms})$ & $\mathrm{ps}$ & $250-375$ \\
Betatron tune $(\mathrm{x}, \mathrm{y})$ & $\cdots$ & $2.177,1.211$ \\
Momentum compaction factor & & 0.244 \\
Beam sizes, x, y $(\sigma)$ & $\mu \mathrm{m}$ & 850,150 \\
Fundamental cavity voltage & $\mathrm{kV}$ & 23 \\
12th harmonic cavity voltage & $\mathrm{kV}$ & 21 \\
\hline \hline
\end{tabular}

signal was split by a mirror that was inserted halfway into the light path. Approximately $1 / 2$ of the signal went to the digital camera and $1 / 2$ to the streak camera.

\section{Optical transport details}

The CMOS digital camera optical path is composed principally of mirrors and focusing lenses. The first mirror in the tunnel collects the full vertical distribution of OSR, with a horizontal and vertical angular coverage of $49 \mathrm{mrad}$. Two filter wheels are used to provide neutral density filters of 0-6 optical density. Optical elements are outlined in Table II below.

The limiting aperture in the CMOS camera optical path is the imaging lens $(f=500 \mathrm{~mm})$. With the optical path in vacuum until the intermediate image, and thereafter in air (of refractive index $n=1$ ), making a thin lens approximation the CMOS camera optical path has a numerical aperture of 0.0053 (accepting $5.3 \mathrm{mrad}$ half angle of synchrotron radiation) in both the horizontal and vertical planes. The optical magnification of the CMOS camera optical path is 0.19 .

The streak camera optical path is composed of several components, principally mirrors and focusing lenses. As per the CMOS camera optical path, neutral density filters of 0-6 optical density are instrumented via two filter wheels.

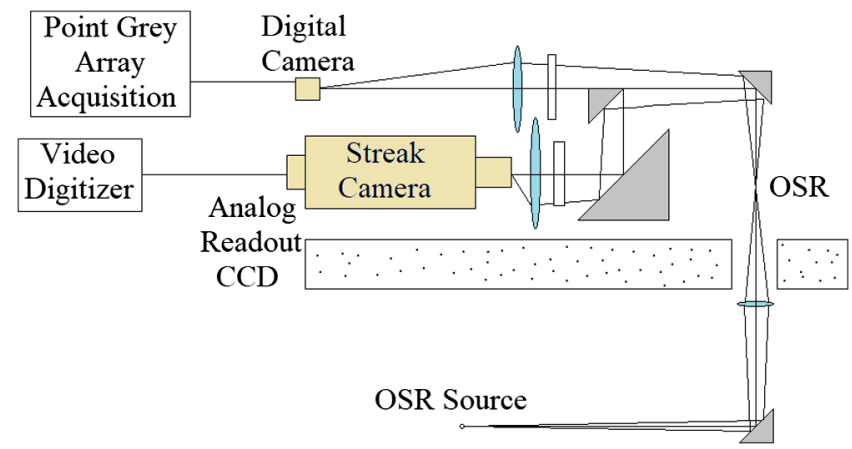

FIG. 2. Schematic illustration of the PAR West optics table with the digital CMOS camera and the streak camera [19].
TABLE II. Optical elements in the CMOS camera path.

\begin{tabular}{lcc}
\hline \hline Element & Location $(\mathrm{m})$ & Aperture $(\mathrm{m})$ \\
\hline Source & 0.00 & $\ldots$ \\
Mirror & 1.62 & 0.10 \\
Mirror & 2.00 & 0.15 \\
Lens $(f=1000 \mathrm{~mm})$ & 2.99 & 0.15 \\
Intermediate image & 4.54 & $\ldots$ \\
Imaging lens $(f=500 \mathrm{~mm})$ & 6.35 & 0.05 \\
Filter wheel & 6.87 & 0.025 \\
Filter wheel & 6.92 & 0.025 \\
Real image $(\mathrm{CMOS}$ camera) & 7.02 & $\ldots$ \\
\hline \hline
\end{tabular}

Additionally, the configuration of mirrors in the optical path introduces rotation of the image. Hence a Dove prism is used to rotate the image $\sim 90^{\circ}$, so that the streak camera can be positioned vertically upright on the optical table and have the horizontal axis along the slit width. Optical elements are outlined in Table III below.

Making a thin lens approximation, the limiting aperture for the streak camera optical path is the Dove prism, giving a numerical aperture of 0.0019 (accepting $1.9 \mathrm{mrad}$ half angle of synchrotron radiation) in both the horizontal and vertical planes. The optical magnification of the streak camera optical path is 0.021 .

\section{Digital CMOS camera}

The 12-bit digital CMOS camera was a FLIR Grasshopper3 USB3 camera with a 1920 by 1200 array of square pixels of 5.86- $\mu \mathrm{m}$ size. It allowed designations of a region of interest (ROI) and output of the integrated counts in combination with the point grey array acquisition. This feature proved to be very useful in tracking the intensity changes over 9 orders of magnitude by adjusting the neutral density (ND) filters (0-6) and sensor integration time $(0.01$ to $10 \mathrm{~s})$ and then tracking electron number and establishing the $\sim 3250$ countsper $\mathrm{e}^{-}$steps in the ROI online for the $375-\mathrm{MeV}$ case. The high quantum efficiency (QE) $(\sim 58 \%$ at $525 \mathrm{~nm})$ for visible wavelengths was advantageous for these studies compared to a CCD camera

TABLE III. Optical Elements in the Streak Camera Path.

\begin{tabular}{lcc}
\hline \hline Element & Location $(\mathrm{m})$ & Aperture $(\mathrm{m})$ \\
\hline Source & 0.00 & $\ldots$ \\
Mirror & 1.62 & 0.10 \\
Mirror & 2.00 & 0.15 \\
Lens $(f=1000 \mathrm{~mm})$ & 2.99 & 0.15 \\
Intermediate image & 4.54 & $\ldots$ \\
Dove prism & 6.50 & 0.015 \\
Filter wheel & 6.87 & 0.025 \\
Filter wheel & 6.92 & 0.025 \\
Imaging lens $(f=1000 \mathrm{~mm})$ & 7.02 & 0.05 \\
Real image $($ Streak camera) & 7.12 & $\ldots$ \\
\hline \hline
\end{tabular}


QE of $\sim 20 \%$. The calibration factor was $31.8 \mu \mathrm{m} /$ pixel (in both $x$ and $y$ axes).

\section{Streak camera}

The Hamamatsu C5680 streak camera included an UVIR transmitting input optics barrel and S20 photocathode. This camera's vertical sweep unit was phase locked to the 117.3 $\mathrm{MHz}$ sine wave input that was amplified in the synchroscan unit to generate the sinusoidal sweep voltages, and the dual axis horizontal sweep unit was installed. This rf source was a fan-out from the master oscillator, and it is the same frequency for the 12th harmonic rf cavity. Arrival times of the photons from the different turns were thus synchronously summed with few-ps jitter, giving the timeaveraged $x$ - $t$ images. The image shape and projections of these two planes provide the unique information on electron OSR emission. The temporal calibration factors on deflection ranges $1-4$ were $0.31,1.05,2.04$, and $2.96 \mathrm{ps} / \mathrm{pixel}$, respectively. The documented temporal resolution of $1.47 \mathrm{ps}$ (FWHM) on the fastest range 1 using monochromatic, 640-nm pulsed laser light was also provided by the vendor data sheet for this M5675 unit [20]. We selected range 4 with its $\sim 1500$ ps time-axis coverage for the bunch-length range expected in the PAR, and the horizontal spatial calibration with our optics was estimated as $150 \mu \mathrm{m} / \mathrm{pixel}$ at the OSR source point by comparison to the image sizes observed in the calibrated CMOS camera. The temporal resolution was $8 \pm 2$ ps $(\sigma)$ with broadband OSR on this range 4 which we used for all tests reported herein. The static spread function contribution was $7.5 \mathrm{ps}$ $(\sigma)$ based on the focus mode profile fit shown in the next section, the phase jitter term was $<2$ ps for the camera in synchroscan mode, and the chromatic temporal dispersion term was $\sim 2.3$ ps for the A1976-01 UV-visible input optics barrel as described previously for the same model optics [21]. These three terms are added in quadrature for the final resolution. This was supported by the observed first-turn image total bunch length of $\sim 10$ ps in the PAR using the PC rf gun micropulse in a parallel experiment with $\sim 4$-ps bunch length due to energy spread and transit time spread in the ring.

\section{EXPERIMENTAL RESULTS}

Initially, we considered a PMT to look for OSR intensity changes at low charge. This proved to be problematic at about $100 \mathrm{fC}$. The 12-bit digital CMOS camera, however, was able to track the average ROI intensity signals down to the few-electron range $(\mathrm{Q} \sim 1 \mathrm{aC})$ and even to a single electron stored with 10 -s sensor integration times. We monitored the integrated counts in the CMOS ROI online as we reduced the stored charge by firing the injection kickers. We typically decreased charge by an order of magnitude and then reduced the ND filter or increased the integration time to compensate. These ratios of background subtracted counts gave us the relative and absolute charges referenced to the initial ICT value at $100 \mathrm{pC}$ down to the 10 -aC level within a factor of 2 , and then we used the electron number in this regime. When we have several electrons only, we also reduce the fundamental rf voltage to obtain single electron loss steps in a more controlled manner. It is the observed discrete and equal steps of 3250 integrated counts for single electron losses or multiples of that number that gave us the absolute charge measurements due to electron number, $N_{e}$. The absolute current value was $1.565 \mathrm{pA} \times N_{e}$. The streak camera data in focus mode and streak mode also showed results at the four- and ten-electron level, respectively, for the first time, to our knowledge.

\section{A. Digital camera data}

The experimental CMOS camera results were exemplified by the recognition of the discrete steps in the intensity as we lost individual electrons as shown in Fig. 3. This plot shows the intensity changes in the ROI of 3250 counts with 10 -s sensor integration time in the 4 to 1 electron (s) stored cases. Insertion of ND 5.0 filters at selected times including at 03:57 and 04:19 checked the baseline which did drift over time. The $1 \mathrm{e}^{-}$intensity level at $04: 25$ is bracketed by the ND test at 04:19 and the purposeful dump of the single electron at $04: 28$. The single electron was stored for $\sim 15$ minutes, or 8.7 billion turns at $375 \mathrm{MeV}$. This is a definitive result, and we acquired similar plots down to a single $\mathrm{e}^{-}$at $425 \mathrm{MeV}$ on other shifts.

Another way to assess the digital CMOS data involved using the images obtained during the run for different electron numbers and subtracting the background image obtained with zero electrons. Examples are shown in Fig. 4 where the background subtracted images for $4,3,2$, and 1

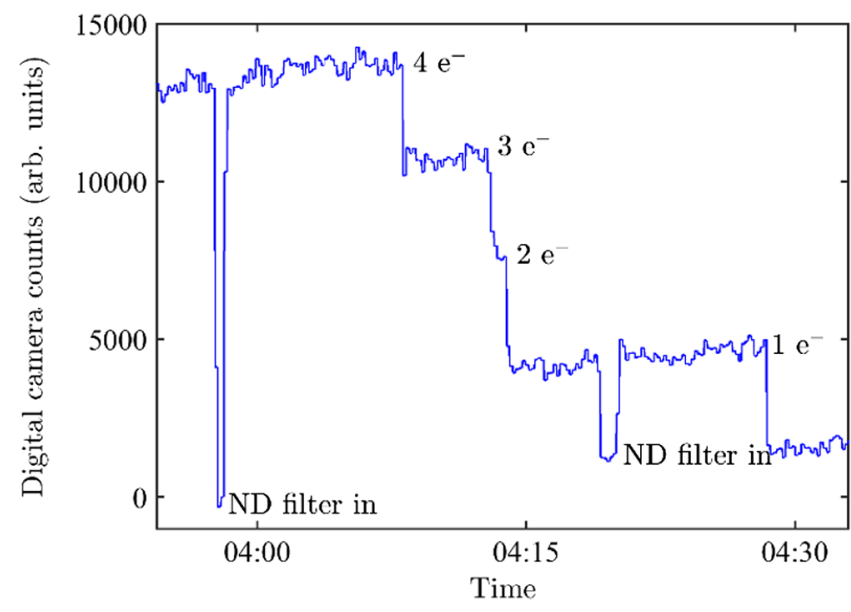

FIG. 3. OSR intensity in the west synchrotron light monitor (PAR:SLM1) versus run-time at 10-s sampling over 35 minutes at $375 \mathrm{MeV}$. The discrete intensity steps are at 3250 counts $/ \mathrm{e}^{-}$. At 04:19, the ND filters were inserted briefly to show the background level while a single electron was stored. This electron was purposely lost at 04:28. 

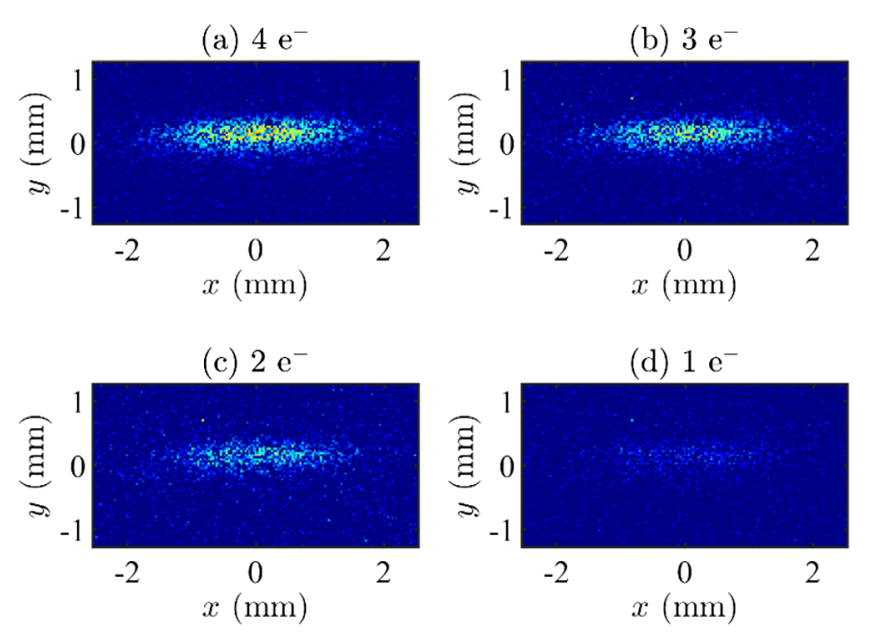

FIG. 4. A set of four CMOS camera 2D images with 10-s sensor integration times. They are background subtracted for the four stored-electron number indicated, 4 to 1 . This is at $375 \mathrm{MeV}$.

electron are shown. The 2D image is shown in all four cases, and the projected $\mathrm{x}$ and $\mathrm{y}$ profile sigma sizes for $4 \mathrm{e}^{-}$ are $838 \pm 6$ and $144 \pm 1 \mu \mathrm{m}$, respectively, based on fits to a single Gaussian profile.

At the limits of the system, we also note that in the streak camera focus mode (where photons are concentrated in a few pixels with the optical minification used in this path) we detected a 2D image with only $4 \mathrm{e}^{-}$stored at $375 \mathrm{MeV}$. This image in Fig. 5 is not with the spatial resolution of the CMOS images in Fig. 4, but with improved optical transport, signal improvements of 4-6 are possible so some increased magnification could be supported. This does illustrate the potential for 2D imaging with OSR at ultralow charges stored using the streak camera's $6 \mathrm{~mm} \times 0.5 \mathrm{~mm}$ photocathode, MCP gain, and electro-optical magnification coupled with image summing (or with a low-noise digital camera with sensor integration time options as the readout camera).

\section{B. Streak camera results}

The streak camera results are partly constrained by the Hamamatsu fiber-optically coupled analog readout CCD camera that was Peltier cooled to $0{ }^{\circ} \mathrm{C}$ to reduce dark current and used with a MaxVideo-200 8-bit video digitizer $(512 \times 480$ array) running at $30 \mathrm{~Hz}$. We acquired a reference nominal-charge streak image with $0.32 \mathrm{nC}$ stored using the streak range with $\sim 1.5$-ns time coverage and $3.0 \mathrm{ps} /$ pixel temporal calibration factor. Figure 6 shows an example focus mode image (a) with a focus $y$ size of $\sigma=$ 2.5 pixels in Fig. 6(b) corresponding to 7.5 ps resolution for monochromatic light with the selected streak range. The entrance slit was set at $1 \mathrm{~mm}$ horizontally by $60 \mu \mathrm{m}$ vertically so the smaller vertical beam size set this streakaxis resolution limit component. Figure 7 shows the streak camera image and a measured bunch length of $372 \pm 12 \mathrm{ps}$ based on a fit to a single Gaussian peak. These are results from the ImageTool MATLAB program [22]. This latter bunch-length value is consistent with the zero-current bunch length for the PAR model at $425 \mathrm{MeV}$ with the 12 th harmonic rf powered on [23-25] and recent photodiode bunch length data [26].

We reduced the stored charge controllably by using the PAR injection kickers down to the single electron circulating. On the way, we obtained hundreds of streak camera images at different charge levels. In Fig. 8(a) we show the accumulated image for $389 \mathrm{e}^{-}$circulating, and in Fig. 8(b) we show the accumulated image for $57 \mathrm{e}^{-}(<9 \mathrm{aC})$ circulating with 200 images summed in both cases and with subtraction of a 200-image sum of the background. The fitted values for a single Gaussian peak are $354 \pm 12$ and $276 \pm 36$ ps for the two cases, respectively.

The high-frequency noise in the images was addressed with a sliding nine-channel average routine with equal weighting. This smoothing then was applied to both the data profile and the summed background image (200 images). It was clear the OSR data were far above the
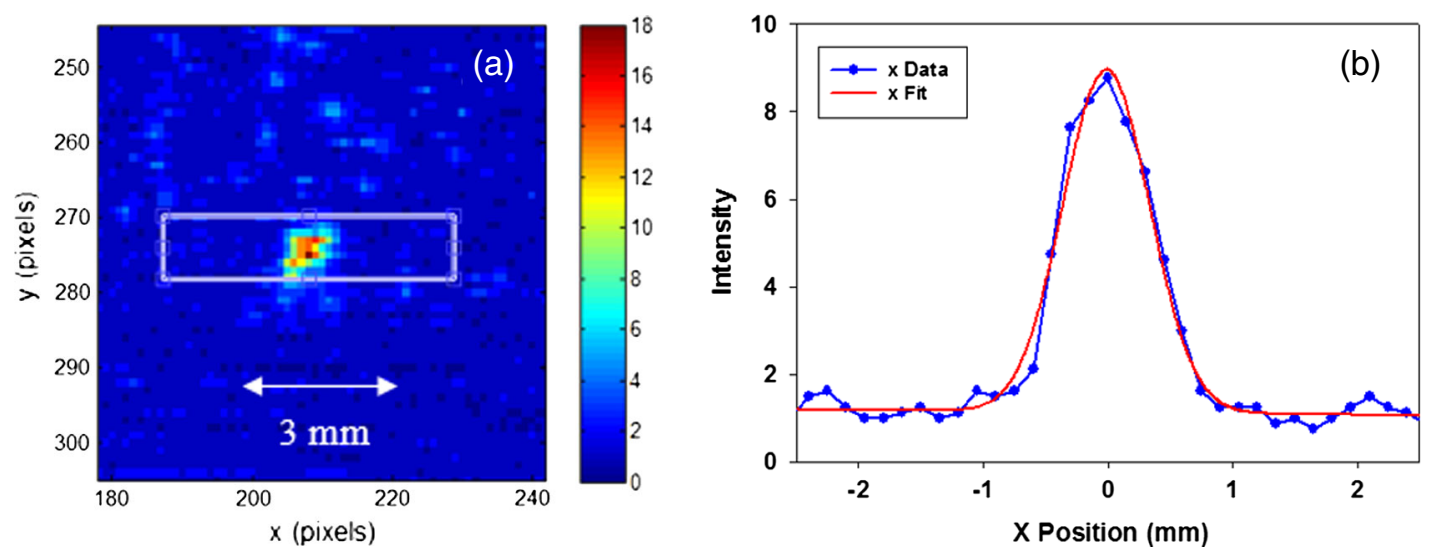

FIG. 5. (a) Streak camera focus mode 50-image sum with median background value subtracted with four electrons stored at $375 \mathrm{MeV}$; (b) projected x profile (blue curve) from the vertically narrowed ROI and the Gaussian profile fit (red curve). A pseudocolor intensity scale is shown at the right of the image. 

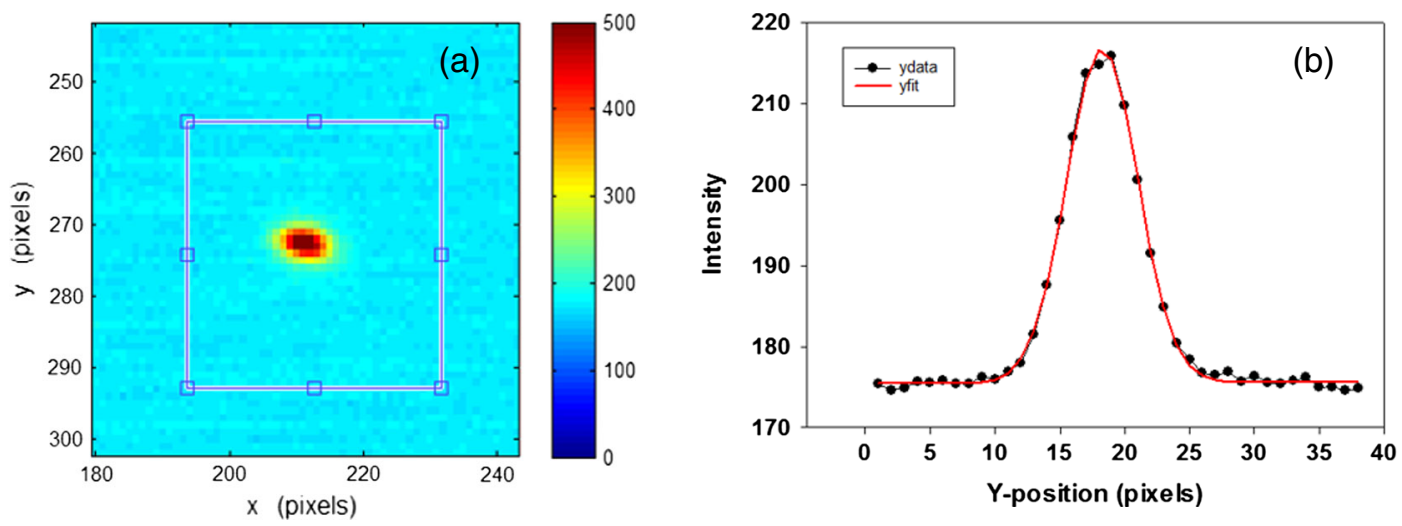

FIG. 6. (a) Focus mode image (sum of 2) and (b) projected y/time-axis profile data (black circles) with single Gaussian profile fit (red) with $\sigma=2.5$ pixels at $0.32 \mathrm{nC}$ and $425 \mathrm{MeV}$. An intensity scale is given in the bar at the right of the image.
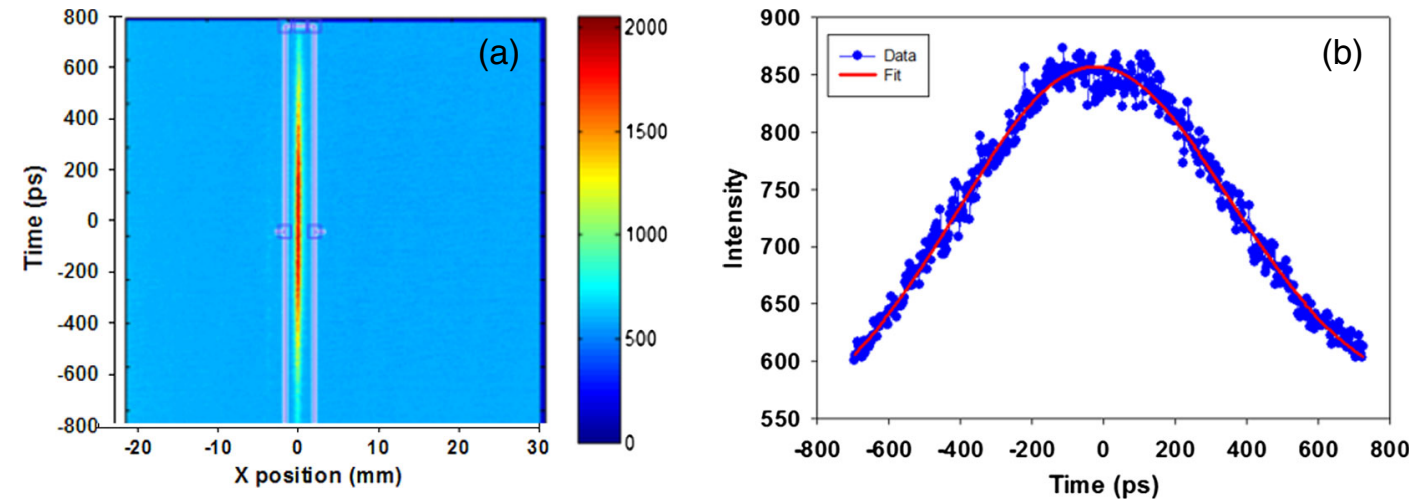

FIG. 7. (a) Streak camera range 4, ten-image sum and (b) time profile (blue) and single Gaussian profile fit (red) with a bunch length of $372 \pm 12 \mathrm{ps}$ at $0.32 \mathrm{nC}$ and with the beam energy of $425 \mathrm{MeV}$. An intensity scale is given in the bar at the right of the image.
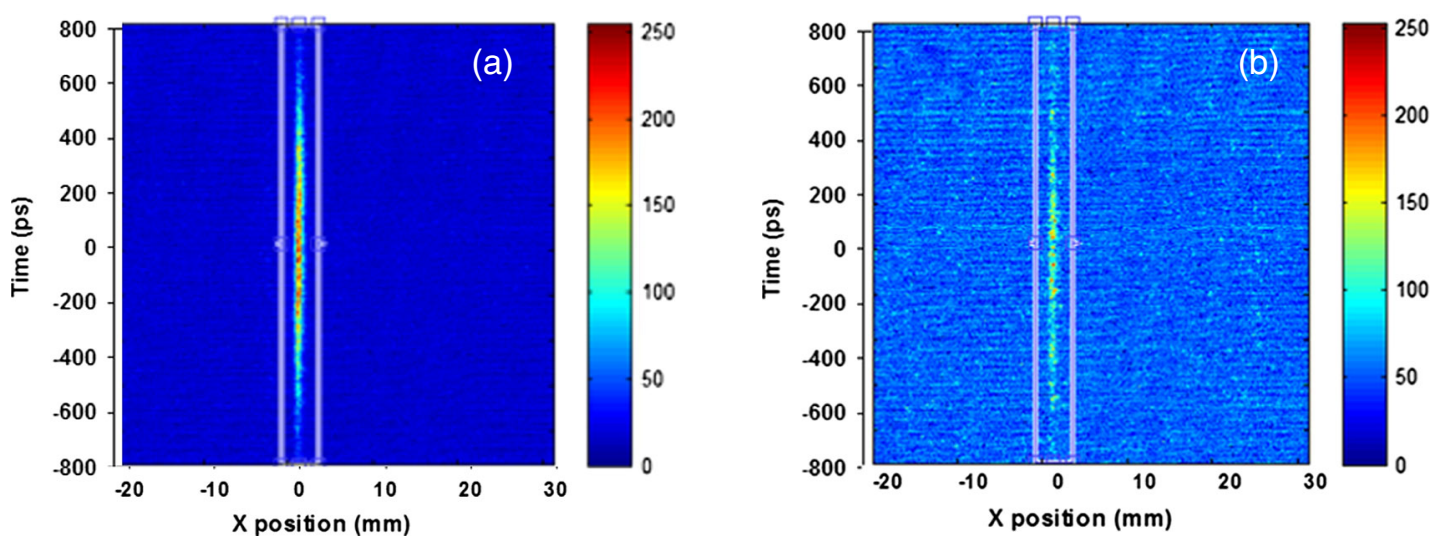

FIG. 8. Streak camera sum image (200 images) at $425 \mathrm{MeV}$ for (a) $389 \mathrm{e}^{-}$and (b) $57 \mathrm{e}^{-}$circulating in the ring. The projected time profile with the single Gaussian peak fit gives the bunch lengths $\sigma_{t}=354 \pm 12$ and $276 \pm 36$ ps, respectively. The digitizer background noise adds to the variance in (b). An intensity scale is given in the bar at the right of each image.

background. The recorded temporal profile is in principle the longitudinal distribution of electrons over $\sim 65$ million turns. In Fig. 9 we explore the temporal distributions at even lower stored electron numbers of 22 and 9 in comparison to the 389 e- data. We observe signals above the background with 200 images summed in all cases. We also note the peak in the $10 \mathrm{e}^{-}$data at the nominal reference particle position at the center of the temporal distribution. 


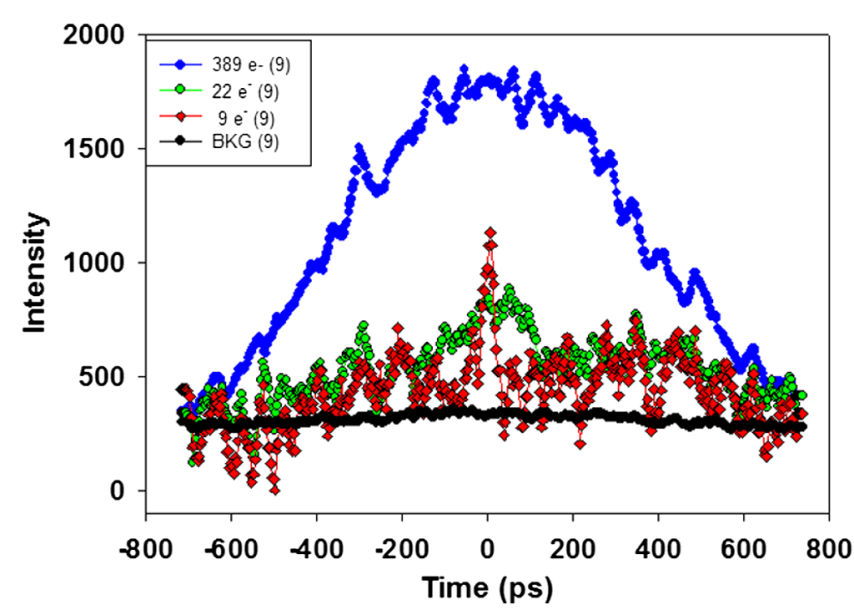

FIG. 9. A comparison of the observed longitudinal distributions for 389,22 , and 9 e- stored with a nine-channel smoothing (9) done on all profiles and 200-image summing used. The ends of the three distributions were matched with the background (BKG) intensity. The central peak near $0 \mathrm{ps}$ in the $10 \mathrm{e}^{-}$case is statistically significant.

We hypothesize that in some cases we may preferentially kick out off-resonance orbit particles with our technique leaving the more resonant orbits. This will be explored in the future using onboard averaging in the digitizer board to reduce the digitizing readout noise role, and improved noise-filtering algorithms will also be considered.

Another example of this diagnostic technique's flexibility and capability is shown in a parallel experiment on the PAR. In this case, the photocathode (PC) rf gun generated a single, short micropulse of $\sim 2$ ps $(\sigma)$ bunch length which was injected after acceleration to $375 \mathrm{MeV}$ into the PAR [27]. Due to energy spread in the bunch, the slightly different orbits and concomitant transit times around the ring led to some lengthening of the distribution

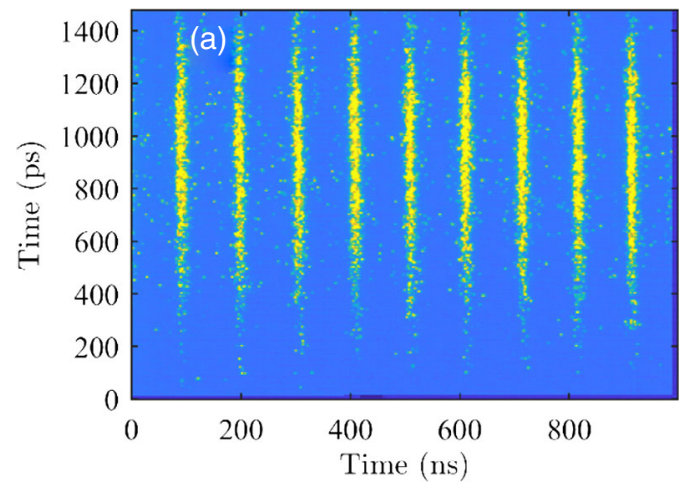

each early turn up to the equilibrium value. The streak camera's vertical sweep was the same synchroscan unit with a span of $1500 \mathrm{ps}$, and the horizontal deflection unit was active and covered $1 \mu$ s as shown in Fig. 10(a). Both rf cavities have power on, and we measure the equilibrium bunch length of $\sim 300 \mathrm{ps}$ in the 14th video frame after injection. The revolution time of $102 \mathrm{~ns}$ is seen in the spacing of the image set. In Fig. 10(b), the PC gun beam was injected with all rf power off, and the first video frame at injection was selected. This initial $\sim 2$-ps bunch length was below the system resolution limit, but it lengthened on the first turn with a total projected temporal profile of $\sigma_{t}=10 \mathrm{ps}$. The system then clearly covers the elongation to $\sim 80 \mathrm{ps}$ in eight turns. Such a system with its $\sim 8$-ps resolution also easily would resolve the reverse case with high sensitivity with OSC predicted to reduce the equilibrium bunch length at $0.1 \mu \mathrm{A}$ from 200 to $\sim 120 \mathrm{ps}$ in IOTA [14] (see our Sec. V). It would even detect much smaller changes in relative bunch length at the $10 \%$ level as one tunes OSC or if some parameters are not optimal.

\section{THE PAR IMPEDANCE MODEL}

The standard impedance model for the PAR was developed over several years [23-25], and this impedance model for the ring was the basis of the calculation of the zero current bunch lengths for stored beam. Input parameters included the fundamental of power at $9.77 \mathrm{MHz}$, the rf power of the 12th harmonic at $117.3 \mathrm{MHz}$, and the impedances of the ring components. The model predicts an increase in the bunch length from 280 to $340 \mathrm{ps}$ with the beam energy change from 375 to $425 \mathrm{MeV}$ for fixed rf power in both cavities and $0.25 \mathrm{nC}$ as shown in Fig. 11. We measured a bunch length of $285 \mathrm{ps}$ at 0.32 -nC stored charge as a reference at $375 \mathrm{MeV}$ and 372 ps at $425 \mathrm{MeV}$ as shown in Fig. 11. The model only predicts minor reductions for charges below $1 \mathrm{pC}$. Normally in the PAR beam damping

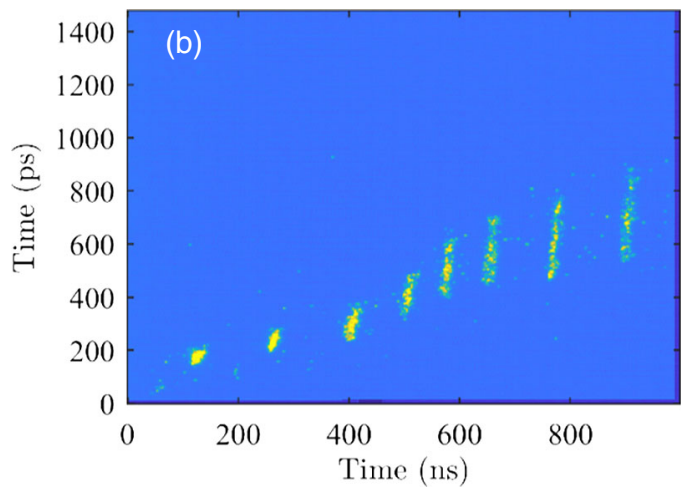

FIG. 10. (a) Dual sweep streak camera image (single frame, 14th after injection trigger) with an equilibrium bunch length of $\sim 300$ ps at $0.5 \mathrm{nC}$ with both rf systems powered on for $375 \mathrm{MeV}$ and (b) dual sweep streak image (first single frame at injection) on the PAR OSR showing the elongation of the bunch over successive turns and the change in arrival time right after initial injection with both rf systems off. The horizontal axis with $1-\mu$ s time range also has some spatial information in a dispersive location at the source where the injection transient shifts the position of the equally spaced turns in time to reflect that spatial transient [27]. A 1- $\mu$ s long MCP gate timed with the horizontal sweep was used for both images. 


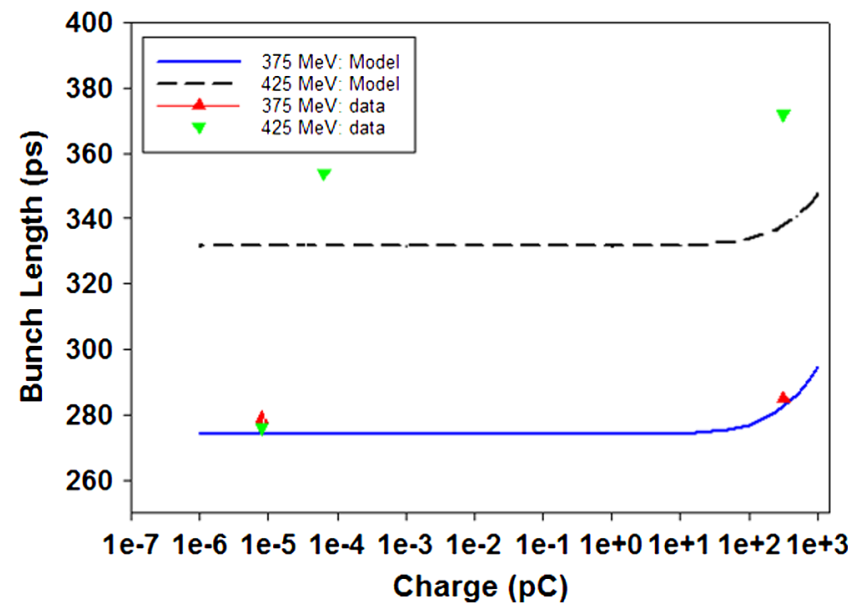

FIG. 11. Predicted equilibrium bunch lengths using the PAR impedance model for a range of charges and the two energies, 375 and $425 \mathrm{MeV}$ [25]. Measured values are also shown as triangles.

cycle, the powering of the 12th harmonic cavity during the cycle reduced the bunch length from $\sim 1 \mathrm{~ns}$ to $\sim 340 \mathrm{ps}$ with $0.5 \mathrm{nC}$ stored. This bunch-length change was detected more than 20 years ago using the streak camera with a slow sweep vertical deflection covering 50-ns full scale and routinely used since then [4]. Our use of the synchroscan unit in the new series of experiments was challenged by bunch lengths longer than $350 \mathrm{ps}$ sigma since the 4-sigma value is $1400 \mathrm{ps}$ and the streak range covered $\sim 1500 \mathrm{ps}$. However, the synchronous summing with reduced phase jitter and improved temporal resolution features were critical and enabling.

\section{POTENTIAL OSC DIAGNOSTICS APPLICATION}

Optical stochastic cooling experiments are an extension to optical frequencies of stochastic cooling experiments for particle beams performed previously in the microwave regime [28]. They are motivated by an increase in the cooling bandwidth by up to 3 orders of magnitude in principle. Complementary experiments on OSC for electrons are being implemented at $1 \mathrm{GeV}$ at the Cornell Electron Storage Ring (CESR) in an arc bypass [29] and at $100 \mathrm{MeV}$ at the Fermilab IOTA ring [14]. This latter experiment will use two undulators (with resonant wavelength of $0.95 \mu \mathrm{m}$ ) in a straight section of the ring for the "pickup undulator" (PU) and the "kicker undulator" (KU). An optical delay path is used to match the transit time of the photon emitted in the PU with the same electron that emitted it (after the transit of a magnetic chicane) in the KU. Small longitudinal kicks to the electrons can result in a cooling effect on the momentum offset with appropriate delay tuning. The predicted bunch-length reduction with OSC in IOTA in the passive mode is from 200 to $120 \mathrm{ps}$ with 83,000 electrons stored $(0.1 \mu \mathrm{A})$. These lower charges are required to avoid the emittance dilution effects of intrabeam scattering that occur at higher charges which could obscure the OSC effects in the transverse plane [14]. In this case the revolution frequency is $7.50 \mathrm{MHz}$ and the synchroscan unit would be tuned to the 11th harmonic at $82.50 \mathrm{MHz}$ [15]. The capabilities of the synchroscan and dual-sweep streak camera techniques could be applied in the following aspects to elucidate the OSC process.

(1) Equilibrium longitudinal distributions.- Such a low charge $\left(83,000 \mathrm{e}^{-}\right)$is still expected to be easily within the expected range for this synchronous summing streak camera technique based on the results reported herein with only 389 electrons stored in a similarly small ring with an effective integration time of 6.6 seconds. In addition, in the Fermilab experiments, a digital readout camera will be used for the streak camera images which would enable summing signals on the camera sensor chip for 1-10 seconds for improved statistics before readout [15]. Sufficient stability of the cooled state is needed. (A preliminary report on first longitudinal distributions measured at IOTA with OSC has been presented by Jarvis and Lebedev [30]).

(2) Transverse equilibrium distributions.-The streak camera focus mode imaging with open slit provides an option for $x-y$ spatial imaging of OSR from a single electron as demonstrated in Fig. 5 at $375 \mathrm{MeV}$. Equilibrium beam x sizes could be measured with an appropriate balance of magnification, stored current, MCP gain, and sensor integration time. The $500-\mu \mathrm{m}$ height of the tube photocathode may limit the $y$-size measurements depending on the beam ellipticity. This could complement the $2 \mathrm{D}$ imaging by a CMOS camera viewing the same scene.

(3) $x$-t effects. - The camera in synchroscan sweep mode also provides the horizontal axis distributions in the same image as shown in Fig. 10 and recent short-range wakefield studies [31]. Optical transport magnifications can be balanced with the stored current to provide enough statistics in the image for complementary equilibrium transverse size with the bunch length. With an OTR source and mirror optics, an effective resolution of $\sim 10 \mu \mathrm{m}$ was indicated at the source point with a spatial calibration factor of $6.6 \mu \mathrm{m} /$ pixel in a similar streak camera system [31]. This was with $\sim 1: 1$ optical transport to the camera and taking advantage of the 3:1 electro-optic magnification within the streak tube from the photocathode to the phosphor. The OSR source is different, but one would expect the diffraction limits are no different than for the CMOS imaging camera being used for the transverse imaging with $\sim 25-\mu \mathrm{m}$ resolution [12].

(4) Cooling rate and T-t effects.-We project that the dual-sweep feature could be applied to the critical question of OSC cooling rate if it were less than 1 second by selecting the $20-, 50$,- or $100-\mathrm{ms}$ horizontal sweep range (T) while the vertical synchroscan unit $(\mathrm{t})$ is still running. The readout camera sensor integration time would need to match the selected $\mathrm{T}$ range and/or the MCP timing gate would be used. However, we start by scaling from the 
$389 \mathrm{e}^{-}$and 6.6-s integration time where the charge stored would need to be about 10,000, 4000, and $2000 \mathrm{x}$ higher, respectively. Intermediate currents of $\sim 25 \mu \mathrm{A}$ should result in equilibrium bunch lengths that fit in the estimated 2.2-ns time window for the $82.50 \mathrm{MHz}$ unit. Otherwise, one could use two different delay settings to shift the streak image to cover the total length in two images or use the slow sweep unit at $\sim 250 \mu \mathrm{A}$ with longer bunches.

If the start of cooling can be controlled and an appropriate trigger provided for the horizontal sweep and the readout camera, then hundreds of dual-sweep images may be acquired and summed to improve statistics and allow using even lower charges or shorter horizontal sweep ranges if warranted. This image summing technique could apply to items (1), (2), and (3) as well to push to ultralow charges and ten electrons or fewer.

\section{SUMMARY}

In summary, we performed initial investigations of OSR emissions from a few electrons stored in the PAR at 375 and $425 \mathrm{MeV}$ with a digital CMOS camera and with a synchroscan streak camera. We were able to identify OSR from a single electron stored using the CMOS camera and OSR from ten electrons stored using a streak camera for the first time. We even detected OSR from four electrons stored in streak camera focus mode and estimate OSR from a single electron could have been detected with a 100-image sum. In addition, we have identified an ideal application of the techniques to an OSC experiment at the Fermilab IOTA ring operating at $100 \mathrm{MeV}$ where a predicted reduction of the equilibrium bunch length by a factor of $40 \%$ with cooling should be detected with high sensitivity by such a system [15]. Additionally, with appropriate setups of the optics and charges, we project one may assess transverse damping at the same time or a subsecond cooling rate for the longitudinal distribution with the dual-sweep technique. These features would be useful for comparison and benchmarking OSC simulations.

\section{ACKNOWLEDGMENTS}

The authors acknowledge the support of J. Byrd and M. Borland of the Accelerator Systems Division and R. Hettel of APS-U as well as the early PAR optical transport and optics table implementation by B. Yang at ANL. A. H. L. acknowledges MATLAB support by R. Thurman-Keup and discussions with V. Lebedev and J. Jarvis (Fermilab) on the OSC simulations and the experiments being planned and now commissioning at IOTA. The submitted manuscript has been created by UChicago Argonne, LLC, Operator of Argonne National Laboratory (Argonne). Argonne, a U.S. Department of Energy Office of Science Laboratory, is operated under Contract No. DE-AC02-06CH11357. The U.S. Government retains for itself, and others acting on its behalf, a paid-up nonexclusive, irrevocable worldwide license in said article to reproduce, prepare derivative works, distribute copies to the public, and perform publicly and display publicly, by or on behalf of the Government.

[1] F. R. Elder, A. M. Gurewitsch, R. V. Langmuir, and H. C. Pollock, Radiation from electrons in a synchrotron, Phys. Rev. 71, 829 (1947).

[2] A. Hoffmann, The Physics of Synchrotron Radiation, Part IV (Cambridge University Press, Cambridge, England, 2004).

[3] A. Lumpkin, RF-synchronized imaging for particle and photon beam characterizations, in Proceedings of the 15th Particle Accelerator Conference, PAC-1993, Washington, DC, 1993 (IEEE, New York, 1993), Vol. 3, pp. 20862090.

[4] B. X. Yang, A. H. Lumpkin, M. Borland, K. Harkay, and A. Nassiri, Characterization of beam dynamics in the APS injector rings using time-resolved imaging techniques, in Proceedings of the Particle Accelerator Conference, Vancouver, BC, Canada, 1997 (IEEE, New York, 1997), pp. 2052-2054.

[5] A. H. Lumpkin and B.X. Yang, Use of Few-Angstrom Radiation Imaging to Characterize Ultrabright, Multi-GeV Particle Beams, Phys. Rev. Lett. 82, 3605 (1999).

[6] J. Hinkson, R. Keller, J. Byrd, and A. Lumpkin, Commissioning of the Advanced Light Source dual-axis streak camera, in Proceedings of the Particle Accelerator Conference, Vancouver, BC, Canada, 1997, paper 6V005, pp. 775-777.

[7] J. Corbett, C. Limborg-Deprey, W. Mok, and A. Ringwall, Commissioning the SPEAR3 diagnostic beam lines, in Proceedings of the 10th European Particle Accelerator Conference, Edinburgh, Scotland, 2006 (EPS-AG, Edinburgh, Scotland, 2006), pp. 1259-1261.

[8] F. Riehle, S. Bernstorff, R. Fröhling, and F. P. Wolf, Determination of electron currents below $1 \mathrm{nA}$ in the storage ring BESSY by measurement of the synchrotron radiation of single electrons, Nucl. Instrum. Methods Phys. Res., Sect. A 268, 262 (1988).

[9] I. V. Pinayev, V. M. Popik, T. V. Shaftan, A. S. Sokolov, N. A. Vinokurov, and P. V. Vorobyov, Experiments with undulator radiation of a single electron, Nucl. Instrum. Methods Phys. Res., Sect. A 341, 17 (1994).

[10] R. Klein et al., Operation of the Metrology Light Source as a primary radiation source standard, Phys. Rev. ST Accel. Beams 11, 110701 (2008).

[11] N. Kuklev, J. D. Jarvis, Y.-K. Kim, A. L. Romanov, J. K. Santucci, and G. Stancari, Synchrotron radiation beam diagnostics at IOTA - commissioning performance and upgrade efforts, in Proceedings of 10th Int. Particle Accelerator Conf. (IPAC'19), Melbourne, Australia, 2019 (JACoW, Geneva, Switzerland, 2019), pp. 27322735, WEPGW103, https://doi.org/10.18429/JACoWIPAC2019-WEPGW103.

[12] G. Stancari, Detecting single electrons in IOTA, Workshop on Single-Electron Experiments in IOTA, Fermilab, Batavia, IL, 2019, https://indico.fnal.gov/event/18395. 
[13] A. H. Lumpkin and K. P. Wootton, Observations of optical synchrotron radiation from ultralow charges stored in a ring operating at $425 \mathrm{MeV}$, in Proceedings of the 9th International Beam Instrumentation Conf. (IBIC'20), Santos, Brazil, 2020 (JACoW, Geneva, Switzerland, 2019), pp. 61-65, https://doi.org/10.18429/JACoW-IBIC2020TUPP18.

[14] V. Lebedev, J. Jarvis, H. Piekarz, A. Romanov, J. Ruan, and M. Andorf, Conceptual Design Report: Optical Stochastic Cooling at IOTA, https://arxiv.org/abs/2012.09967.

[15] A. H. Lumpkin, J. Jarvis, and V. Lebedev, Proposed longitudinal profile diagnostics for optical stochastic cooling of stored electrons in the IOTA ring (to be published).

[16] C.-Y. Yao and E. Norum, Bunch current and phase detection for the APS PAR, in Proceedings of the 23rd Particle Accelerator Conference, Vancouver, Canada, 2009 (IEEE, Piscataway, NJ, 2009) TH5RF006, pp. 3447-3449.

[17] A. R. Brill, J. R. Calvey, K. C. Harkay, R. T. Keane, N. Sereno, U. Wienands, K. P. Wootton, and C. Yao, Current monitor and beam position monitor performance for high charge operation of the Advanced Photon Source particle accumulator ring, in Proceedings of the 8th International Beam Instrumentation Conference 2019 (IBIC'2019), Malmo, Sweden, 2019 (JACoW, Geneva, Switzerland, 2019), WEPP017, pp. 552-554, https://doi.org/10.18429/ JACoW-IBIC2019-WEPP017.

[18] S. Shin, Y. Sun, J. Dooling, M. Borland, and A. Zholents, Interleaving lattice for the Argonne Advanced Photon Source linac, Phys. Rev. Accel. Beams 21, 060101 (2018).

[19] W. Berg, B. Yang, A. Lumpkin, and J. Jones, Design and commissioning of the photon monitors and optical transport lines for the Advanced Photon Source positron accumulator ring, AIP Conf. Proc. 390, 483 (1997).

[20] Hamamatsu Photonics Data Sheets for Model C5680 Streak Camera and Vertical plugin units. Calibrations and resolution test performed at the factory based on $640 \mathrm{~nm}$ wavelength of a fast laser pulse used with a calibrated etalon (unpublished).

[21] A. H. Lumpkin, J. Ruan, and R. M. Thurman-Keup, Synchroscan streak camera imaging at a $15-\mathrm{MeV}$ photoinjector with emittance exchange, Nucl. Instrum. Methods Phys. Res., Sect. A 687, 92 (2012).

[22] R. Thurman-Keup, A. S. Johnson, A. H. Lumpkin, and J. Ruan, Transverse emittance and phase space program developed for use at the Fermilab A0 photoinjector, in Proceedings of the 24th Particle Accelerator Conference, PAC-2011, New York, 2011 (IEEE, New York, 2011), pp. 528-530.

[23] M. Borland, Update on the Argonne Positron Accumulator Ring, in Proceedings of the 15th Particle Accelerator Conference, PAC-1993, Washington, DC, 1993 (Ref. [4]), pp. 2028-2030.

[24] C.-Y. Yao, B. Yang, and H. Shang (private communication).

[25] K. Harkay (private communication).

[26] J. C. Dooling, J. R. Calvey, K. C. Harkay, B. X. Yang, and C. Yao, Fast photodetector bunch duration monitor for the Advanced Photon Source particle accumulator ring, in Proceedings of 9th Int. Particle Accelerator Conf. (IPAC'18), Vancouver, Canada (JACoW, Geneva, Switzerland, 2018), pp. 1819-1822, https://doi.org/10.18429/ JACoW-IPAC2018-WEPAF006.

[27] K. P. Wootton, J. R. Calvey, J. C. Dooling, K. C. Harkay, Y. Sun, B.X. Yang, and A. H. Lumpkin, Streak camera measurement of electron beam energy loss per turn in the advanced photon source particle accumulator ring, in 9th Int. Beam Instrumentation Conf. (IBIC'20), Santos, Brazil (JACoW, Geneva, Switzerland, 2020), pp. 66-69, https://doi.org/10.18429/JACoW-IBIC2020-TUPP23.

[28] D. Moehl, G. Petrucci, L. Thorndale, and S. van der Meer, Physics and technique of stochastic cooling, Phys. Rep. 58, 73 (1980).

[29] M. B. Andorf, W. F. Bergan, I. V. Bazarov, J. M. Maxson, V. Khachatryan, D. L. Rubin, and S. T. Wang, Optical stochastic cooling with an arc bypass in the Cornell Electron Storage Ring, Phys. Rev. Accel. Beams 23, 102801 (2020).

[30] J. Jarvis and V. Lebedev, OSC at IOTA: The world's first experimental demonstration of optical stochastic cooling, at the Fermilab Accelerator Physics and Technology Seminar, May 11, 2021.

[31] A. H. Lumpkin, R. M. Thurman-Keup, D. Edstrom, and J. Ruan, Submicropulse electron-beam dynamics correlated with short-range wakefields in Tesla-type superconducting rf cavities, Phys. Rev. Accel. Beams 23, 054401 (2020).

Correction: Reference citation errors in the first sentence of Sec. IV and in the caption to Fig. 11 have been fixed. 